\title{
ON A MATHEMATICAL MODEL FOR AN OLD CARD TRICK
}

\author{
Roy Quintero \\ Department of Mathematics, University of Iowa, USA \\ roy-quintero@uiowa.edu
}

\begin{abstract}
The three-pile trick is a well-known card trick performed with a deck of 27 cards which dates back to the early seventeenth century at least and its objective is to uncover the card chosen by a volunteer. The main purpose of this research is to give a mathematical generalization of the three-pile trick for any deck of $a b$ cards with $a, b \geqslant 2$ any integers by means of a finite family of simple discrete functions. Then, it is proved each of these functions has just one or two stable fixed points. Based on this findings a list of 222 (three-pile trick)-type brand new card tricks was generated for either a package of 52 playing cards or any appropriate portion of it with a number of piles between 3 and 7 . It is worth noting that all the card tricks on the list share the three main properties that have characterized the three-pile trick: simplicity, self-performing and infallibility. Finally, a general performing protocol, useful for magicians, is given for all the cases. All the employed math techniques involve naive theory of discrete functions, basic properties of the quotient and remainder of the division of integers and modular arithmetic.
\end{abstract}

Keywords: Modular arithmetic, fixed point theory of discrete functions, three-pile trick.

\section{Introduction}

Among other reasons, the three-pile trick (TPT) is preferred by mathematicians because of its infallibility and simplicity. The origin of this interesting card trick goes back around four centuries [1]. According to Gardner [6] - for a pack of 27 cards - it is one of the oldest of mathematical tricks that involve the ordering of cards, and one of the most intriguing. Probably, the first person to whom the TPT was tractable in a formal way - mathematically speaking - was Joseph Diez Gergonne [7, a French geometer from the nineteenth century. He generalized the trick for any deck of $m^{m}$ cards ( $m>1$ any integer) by applying some combinatorial ideas. Additional papers were written along that century 
incorporating new results [5, 9, but in all of them the technique employed was similar to Gergonne's. During the past century the trick was included in books on recreational mathematics, the main are by Ball and Coxeter [2], Hugard [10, and Gardner [6]. It might sound strange saying that only a few mathematical advances on the problem and its generalization were achieved in the twentieth century. But lately the TPT has recovered its prominence as a math object in various contexts. For instance, it has been treated in an algorithmic manner [12], also as a numerical process in connection with the positional notation system [3, 11], and as a discrete dynamical system [4, 8] too. In this note the trick is seen as a simple function from a finite set of integers to itself.

\section{The TPT and its generalization}

Let us begin by introducing some basic notation. Given any pair of nonnegative integers $j \leqslant k$ the set of natural numbers $\{j, \ldots, k\}$ is denoted by $[j: k]$. The symbols $q(x, a)$ and $r(x, a)$ represent the quotient and the remainder respectively when the integer $x$ is divided by the nonzero integer $a$. Given any function $D \stackrel{f}{\rightarrow} D$, the composite function $f \circ \cdots \circ f$ ( $n$ times) is denoted by $f^{n}$ and the set of its fixed points by Fix $(f)$, i.e., Fix $(f)=\{x \in D \mid f(x)=x\}$.

As it was established in [11, given the $c^{\text {th }}$ card (counting from the top) in a deck of 27 cards face down is said that the TPT is performed on it, if the function $G_{2}:[1: 27] \rightarrow[1: 27]$ defined by $x \mapsto q(x, 3)+9+\operatorname{sign}(r(x, 3))$ is composed three times at $x=c$.

Before continuing with our presentation, let us figure out why the algebraic expression $q(x, 3)+9+\operatorname{sign}(r(x, 3))$ encodes what is happening with the TPT. In fact, a simple example will make it clear. Suppose that the chosen card is the 19th (counting from the top). Well, $q(19,3)=6$ gives us the number of cards below it after dealing the cards in three piles when the TPT is performed. So, after collecting the three piles the 19th card will occupy the 16th position (remember that piles while collecting are all flipped over) which is equal to the sum of $6(=q(19,3)), 9$ (the cards of the pile placed at the top) and 1 $(=\operatorname{sign}(r(19,3)))$.

To precise more the notation employed, the sub-index 2 in $G_{2}$ indicates that after dealing the whole deck of 27 cards the pile containing the chosen card will be placed in the middle of the other two but this is equivalent to say that this particular pile is the second one counting from the bottom after collecting the three piles face down.

Two important properties of this discrete function are:

1. Its only fixed point is 14 (i.e., $F i x\left(G_{2}\right)=\{14\}$ ).

2. The composite function $G_{2}^{3} \equiv 14$ (i.e., $G_{2}^{3}(x)=14$ for every $x \in[1: 27]$ ).

Remark 1. What makes the TPT an infallible card trick is property 2. Also 
observe one has to apply the same procedure three times and this is what makes the trick easy to perform and impressive in some sense, hence its simplicity.

Let us show how the TPT should be generalized in a natural way. To start just assume that our deck has $a b$ cards (face down). If we apply the trick with $a$ piles, then each will have $b$ cards. Of course the trick is trivial when $a, b=1$. From now on, let us assume $a, b \geqslant 2$. In [11], the general case under study was also considered and the appropriate function for a deck of $a b$ cards is $G_{p}:[1: a b] \rightarrow[1: a b]$ defined by $x \mapsto q(x, a)+b(p-1)+\operatorname{sign}(r(x, a))$ for every $p \in[1: a]$. In this general setting, the value $p$ indicates that when the new trick is performed the pile with the chosen card will be placed in the $p$-th position counting from the bottom once the $a$ piles are being recollected.

Our first mathematical result is Lemma 2 which gives a list of relevant properties of the functions $G_{1}, \ldots, G_{a}$.

Lemma 2. Let $a, b \geqslant 2$ be fixed integers. The finite family of functions $\left\{G_{p}\right\}_{p=1}^{a}$ satisfies the following properties:

1. The function $G_{p}$ is nondecreasing for every $p$.

2. $\operatorname{Im}\left(G_{p}\right)=[b(p-1)+1: b p]$ for every $p$.

3. If $p_{1}<p_{2}$, then $G_{p_{1}}<G_{p_{2}}$.

4. The function $G_{1}$ has 1 as its unique fixed point, i.e., Fix $\left(G_{1}\right)=\{1\}$.

5. The function $G_{a}$ has ab as its unique fixed point, i.e., Fix $\left(G_{a}\right)=\{a b\}$.

6. Assume $a \geqslant 3$. For each $p \in[2: a-1]$ holds:

(a) If $a-1 \mid b(p-1)$, then Fix $\left(G_{p}\right)=\left\{x_{p, 1}, x_{p, 1}+1\right\}$ where

$$
x_{p, 1}=\frac{a b(p-1)}{a-1} \text {. }
$$

(b) If $a-1 \backslash \backslash b(p-1)$, then Fix $\left(G_{p}\right)=\left\{x_{p, 2}\right\}$ where

$$
x_{p, 2}=\frac{a b(p-1)+a-j(p)}{a-1}
$$

being $j(p)$ the unique integer in $[2: a-1]$ that is solution of the modular equation $X \equiv a b(p-1)+a \bmod (a-1)$.

Proof. 1. Let $2 \leq p \leq a$. From the definitions of $G_{1}$ and $G_{p}$ follows the relationship $G_{p}(x)=G_{1}(x)+b(p-1)$ for every $x \in[1: a b]$. So, we only need to prove that $G_{1}$ is nondecreasing. Set $I_{k}=[(k-1) a+1: k a]$ for $k=1, \ldots, b$. Clearly, the finite class $\left\{I_{1}, \ldots, I_{b}\right\}$ is a partition of $[1: a b]$. By observing carefully the behavior of $G_{1}$ on each interval of the partition we have $\operatorname{Im}\left(G_{1 \mid I_{k}}\right)=\{k\}$ for $k=1, \ldots, b$, and from this follows that $G_{1}$ is certainly nondecreasing. 
2. By the latter condition $G_{1}$ maps $[1: a b]$ on $[1: b]$ and from this we get the expected result.

3. It follows immediately from property 2 .

4. Let us see that 1 is fixed by $G_{1}$. In fact, $G_{1}(1)=q(1, a)+b(1-1)+$ $\operatorname{sign}(r(1, a))=0+0+1=1$. Suppose now that $x \in[1: a b]$ is any fixed point of $G_{1}$. Hence $x$ satisfies $q(x, a)+\operatorname{sign}(r(x, a))=x$. Observe that $r(x, a)$ has to be nonzero. Otherwise, $q(x, a)=0$, and $x=0$ which is a contradiction. Then, it holds $q(x, a)=\frac{1-r(x, a)}{a-1}$, but $q(x, a) \geqslant 0$ and $-1<\frac{1-r(x, a)}{a-1} \leqslant 0$. So, $r(x, a)=1$, and $x=q(x, a) a+r(x, a)=1$. Therefore, Fix $\left(G_{1}\right)=\{1\}$.

5. First, $a b$ is a fixed point of $G_{a}$ since $G_{a}(a b)=q(a b, a)+b(a-1)+\operatorname{sign}(r(a b, a))=$ $b+b(a-1)+0=a b$. Now, suppose $x \in[1: a b]$ is any fixed point of $G_{a}$. Hence $x$ satisfies $q(x, a)+b(a-1)+\operatorname{sign}(r(x, a))=x$. We claim that $r(x, a)=0$. Otherwise, by writing $x=q(x, a) a+r(x, a)$ in the latter equation and solving for $b-q(x, a)$ follows $b-q(x, a)=\frac{r(x, a)-1}{a-1}$, but $b-q(x, a)$ is a nonnegative integer and $0 \leqslant \frac{r(x, a)-1}{a-1}<1$. So, $r(x, a)=1, q(x, a)=b$, and $x=a b+1$ which is a contradiction. Then, it holds $(b-q(x, a))(a-1)=0$. Thus, $q(x, a)=b$, and $x=a b$. Therefore, $\operatorname{Fix}\left(G_{a}\right)=\{a b\}$.

6. (a) By hypothesis, $x_{p, 1}$ is well defined. Since $0<a b(p-1) /(a-1)<a b$, $x_{p, 1} \in[1: a b-1]$ and $x_{p, 1}+1 \in[2: a b]$. So, both values are included in the domain of $G_{p}$. Now observe that

$$
\begin{aligned}
G_{p}\left(x_{p, 1}\right) & =q\left(x_{p, 1}, a\right)+b(p-1)+\operatorname{sign}\left(r\left(x_{p, 1}, a\right)\right) \\
& =\frac{b(p-1)}{a-1}+b(p-1)+0=x_{p, 1}
\end{aligned}
$$

and

$$
\begin{aligned}
G_{p}\left(x_{p, 1}+1\right) & =q\left(x_{p, 1}+1, a\right)+b(p-1)+\operatorname{sign}\left(r\left(x_{p, 1}+1, a\right)\right) \\
& =\frac{b(p-1)}{a-1}+b(p-1)+1=x_{p, 1}+1 .
\end{aligned}
$$

Thus, both points are fixed by $G_{p}$. Next, assume that $x \in[1: a b]$ is any fixed point of $G_{p}$. We claim that either $r(x, a)=0$ or $r(x, a)=1$. Otherwise, $2 \leqslant r(x, a) \leqslant a-1$ and $x$ satisfies $q(x, a)+b(p-1)+1=x$. Multiply the latter equation by $a$ and express $q(x, a) a=x-r(x, a)$ in order to get $a b(p-1)+a-r(x, a)=x(a-1)$ which indicates that $a-1 \mid a b(p-1)+a-r(x, a)$, but $a-1 \mid a b(p-1)$. Then, $a-1 \mid a-r(x, a)$ an impossibility because $1 \leqslant a-r(x, a) \leqslant a-2$. So, $r(x, a) \in\{0,1\}$ as claimed. The first case makes $x=x_{p, 1}$ and the second $x=x_{p, 1}+1$. Therefore, $\operatorname{Fix}\left(G_{p}\right)=\left\{x_{p, 1}, x_{p, 1}+1\right\}$. This proves $6(\mathrm{a})$.

(b) Suppose that $x \in[1: a b]$ were a fixed point of $G_{p}$. If $r(x, a)=0$ or $r(x, a)=1$, then $x$ should satisfy $q(x, a)+b(p-1)=q(x, a) a$. From which we get $b(p-1)=q(x, a)(a-1)$. In other words, $a-1 \mid b(p-1)$ contradicting the hypothesis. Therefore, $r(x, a)$ must belong to $\{2, \ldots, a-1\}$, and must satisfy $q(x, a)+b(p-1)+1=x$. Multiply the latter equation by $a$ and replace $q(x, a) a$ 
by $x-r(x, a)$ to get $a b(p-1)+a-r(x, a)=x(a-1)$. In other words, $r(x, a)$ is congruent to $a b(p-1)+a$ modulo $a-1$, i.e., $r(x, a)$ is the unique solution of equation $X \equiv a b(p-1)+a \bmod (a-1)$. Let $j(p)$ be this solution. Thus, the value $x_{p, 2}$ is well defined and $x=x_{p, 2}$. Moreover, $j(p)=r\left(x_{p, 2}, a\right)$ and

$$
\begin{aligned}
G_{p}\left(x_{p, 2}\right) & =q\left(x_{p, 2}, a\right)+b(p-1)+\operatorname{sign}\left(r\left(x_{p, 2}, a\right)\right) \\
& =\frac{1}{a}\left[x_{p, 2}+a b(p-1)+a-j(p)\right]=\frac{1}{a}\left[x_{p, 2}+x_{p, 2}(a-1)\right]=x_{p, 2},
\end{aligned}
$$

as expected, and this completes the proof of $6(\mathrm{~b})$.

An immediate consequence of Lemma 2 is the following corollary.

Corollary 3. Let $a \leqslant 3$ and $n \leqslant 2$ be fixed integers. If $b=a^{n-1}$, then Fix $\left(G_{p}\right)=\left\{x_{p, 2}\right\}$ for every $p \in[2, a-1]$.

Proof. It follows from the fact $a-1 \nmid a^{n-1}(p-1)$ for every $p \in[2: a-1]$.

In 4, Theorem 3] the TPT was generalized for a deck of $p q$ cards where $p, q \geq 3$ are any odd integers through a function denoted by $h$ and proved that $\frac{p q+\overline{1}}{2}$ is a fixed point of $h$. Well, that result also follows from Lemma 2 after making some easy symbolic translations.

Corollary 4. Theorem 3 given in [4] holds.

Proof. Before starting the proof we need to translate our notation to Champanerkar and Jani's. Well, $a=q, b=p$, and $G_{\frac{q+1}{2}}=h$. When $p, q \geq 3$ are odd numbers then $q-1 \backslash p\left(\frac{q+1}{2}-1\right)$. Otherwise $p$ would be an even integer. By Lemma 2.6(b), we have to find the unique fixed point $x_{\frac{q+1}{2}, 2}$ of $h$ by solving first the modular equation $X \equiv p q(q-1) / 2+q \bmod (q-1)$ in $[2: q-1]$. Since $(p q(q-1) / 2+q)-(q+1) / 2=((p q+1) / 2)(q-1)$ the solution searched for is $j\left(\frac{q+1}{2}\right)=\frac{q+1}{2}$. Then,

$$
x_{\frac{q+1}{2}, 2}=\frac{p q+1}{2}
$$

as expected.

When dealing the cards for the first time-providing that $a-1 \mid b(p-1)$ $(2 \leq p \leqslant a-1)$-the values $x_{p, 1}$ and $x_{p, 1}+1$ always fall in the $a^{\text {th }}$ pile and the $1^{s t}$ pile respectively. By taking $s=\frac{b(p-1)}{a-1} \in[1: b], x_{p, 1}=s a$ and $x_{p, 1}+1=s a+1$, both are indicated inside a box in Table 1. Fixed points of type $x_{p, 2}(2 \leqslant p \leqslant a-1)$ only appear at intermediate columns and can not be located at the $1^{\text {st }}$ and $b^{\text {th }}$ rows. Let us see the examples $a=4, b=6$ and $a=3$, $b=5$.

Example 5. Since $3 \mid 6$ we need to apply equation (II). So, $x_{2,1}=8$ and $x_{3,1}=16$. Hence Fix $\left(G_{2}\right)=\{8,9\}$ and Fix $\left(G_{3}\right)=\{16,17\}$ (Figure 1(a)). 
Table 1: First dealing

$\begin{array}{ccccccc}\text { pile } 1 & \text { pile } 2 & \ldots & \text { pile } j & \ldots & \text { pile } a-1 & \text { pile } a \\ (b-1) a+1 & (b-1) a+2 & \ldots & (b-1) a+j & \ldots & (b-1) a+a-1 & b a \\ \vdots & \vdots & \vdots & \vdots & \vdots & \vdots & \vdots \\ s a+1 & s a+2 & \ldots & s a+j & \ldots & s a+a-1 & (s+1) a \\ (s-1) a+1 & (s-1) a+2 & \ldots & (s-1) a+j & \ldots & (s-1) a+a-1 & s a \\ \vdots & \vdots & \vdots & \vdots & \vdots & \vdots & \vdots \\ a+1 & a+2 & \ldots & a+j & \ldots & a+a-1 & 2 a \\ 1 & 2 & \ldots & j & \ldots & a-1 & a\end{array}$

Example 6. Since $2 \nmid 5$ we need to solve the modular equation $X \equiv 18 \bmod 2$ whose unique solution inside interval [2:2] is $j(2)=2$. Then, by applying formula (21), we get $x_{2,2}=8$. Hence Fix $\left(G_{2}\right)=\{8\}$ (Figure 1(b)).

From Examples $\left[\right.$ and $\left[\right.$ lone might ask whether the finite class $\left\{\text { Fix }\left(G_{p}\right)\right\}_{p=2}^{a-1}$ is always formed by sets of one element or by sets of two elements. Next example shows that it can be mixed.

Example 7. Take $a=5, b=2$ (Figure 1(c)). Then Fix $\left(G_{2}\right)=\{3\}$, Fix $\left(G_{3}\right)=$ $\{5,6\}$, and Fix $\left(G_{4}\right)=\{8\}$.

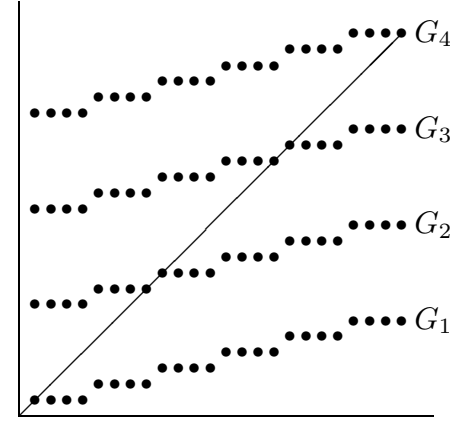

(a)

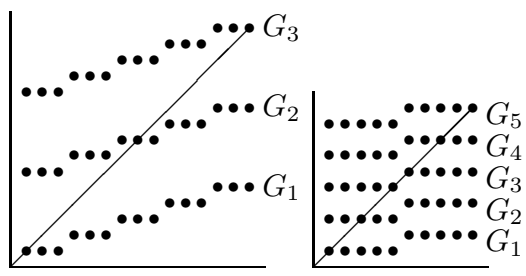

(c)

Figure 1: Examples 5, 6, and 7.

\section{Main results}

In this section we prove some important facts of the family of functions $\left\{G_{p}\right\}_{p=1}^{a}$ in terms of discrete dynamical systems. The first result generalizes the TPT because in this case $b=9$ is a power of $a=3$. Theorem $8(\mathrm{~b})$ shows that $x_{p, 2}$ is an attractor and this is the condition that makes the TPT work. 
Let us fix more notation. Let $A, B, C$ and $D$ denote the sets $[2: a-1],[1: a b]$, $\left[1: x_{p, 1}\right]$, and $\left[x_{p, 1}+1: a b\right]$ respectively. Set $y_{n}:=\frac{a^{n}-1}{a-1}$ for every $n \geqslant 2$.

Theorem 8. If $p \in A$ and $b=a^{n-1}$, then

(a) $x_{p, 2}=y_{n}(p-1)+1$, and

(b) $G_{p}^{n}(x)=x_{p, 2}$ for every $x \in B$.

Proof. (a) Observe that

$$
a^{n}(p-1)+a-p=\left(a^{n}-1\right)(p-1)+(a-1)=\left(y_{n}(p-1)+1\right)(a-1) .
$$

Thus, by Lemma [2] 6(b), $x_{p, 2}=y_{n}(p-1)+1$.

(b) By [11, Théorème II.2.4.] we have

$$
G_{p}^{n}(x)=(p-1) a^{n-1}+\cdots+(p-1) a+(p-1)+1,
$$

but the latter value coincides with $y_{n}(p-1)+1$.

By taking $a=3$ and $b=9$ in Theorem $\left[\right.$ we get $x_{2,2}=y_{3}+1=14$ and $G_{2}^{3}(x)=14$ for every $x \in[1: 27]$. In other words, the TPT is checked.

The following theorem constitutes the most important result of this research. Theorem 8(b) and Theorem 9 guarantee that all the fixed points obtained in Lemma 2 parts 4,5 and 6 are attractors. Based on this property we will be able to extend - in the next section - the TPT by listing more than 200 new variants of the trick by varying the number of cards in the deck, the number of piles and the performance protocol.

Theorem 9. Let $a \geqslant 3, b \geqslant 2$ and $n \geqslant 2$ be fixed integers. If $p \in[1: a]$ and $a^{n-2}<b<a^{n-1}$, then

$$
\begin{aligned}
& G_{p}^{n}(x)= \begin{cases}1 & \text { if }(p, x) \in\{1\} \times B \\
x_{p, 1} & \text { if }(p, x) \in A \times C, a-1 \mid b(p-1), b \neq a^{n-2}+1 \\
x_{p, 1}+1 & \text { if }(p, x) \in A \times D, a-1 \mid b(p-1), b \neq a^{n-2}+1 \\
x_{p, 2} & \text { if }(p, x) \in A \times B, a-1 \nmid \backslash b(p-1), b \leq \frac{a^{n-1}}{a-2} \\
a b & \text { if }(p, x) \in\{a\} \times B\end{cases} \\
& G_{p}^{n-1}(x)= \begin{cases}x_{p, 1} & \text { if }(p, x) \in A \times C, a-1 \mid b(p-1), b=a^{n-2}+1 \\
x_{p, 1}+1 & \text { if }(p, x) \in A \times D, a-1 \mid b(p-1), b=a^{n-2}+1\end{cases}
\end{aligned}
$$

and for $a>3$

$$
G_{p}^{n+1}(x)=x_{p, 2} \quad \text { if }(p, x) \in A \times B, a-1 \nmid b(p-1), b>\frac{a^{n-1}}{a-2} .
$$


Proof. Equations (Bai) and (Bel) were proved in [11, Théorème II.2.1.] and [11, Théorème II.2.2.] respectively. For the remaining six parts we use the same technique employed in [11].

For (3b), take $x \leqslant x_{p, 1}$ arbitrary, say, $x=(s-1) a+j$ as is shown in Table 1, then $s \leqslant \frac{b(p-1)}{a-1}=\frac{x_{p, 1}}{a}$. From [11, Théorème II.1.] it follows that

$$
A(s) \leqslant G_{p}^{n}(x) \leqslant B(s),
$$

where $A(s)=\frac{1}{a^{n-1}}\left(\frac{x_{p, 1}}{a}\left(a^{n}-1\right)+s\right)$ and $B(s)=A(s)+1-\frac{1}{a^{n-1}}$.

Our next goal is to demonstrate the four inequalities

$$
x_{p, 1}-1<A(s) \leqslant x_{p, 1} \leqslant B(s)<x_{p, 1}+1
$$

for every $s \leqslant a^{-1} x_{p, 1}$. After doing many calculations and simplifications we get

$$
\left\{\begin{array}{lll}
x_{p, 1}-1<A(s) & \Longleftrightarrow \quad x_{p, 1}<a^{n}+s a \\
A(s) \leq x_{p, 1} & \Longleftrightarrow \quad x_{p, 1} \geq s a \\
x_{p, 1} \leq B(s) & \Longleftrightarrow \quad x_{p, 1} \leq a^{n}+s a-a \\
B(s)<x_{p, 1}+1 & \Longleftrightarrow \quad x_{p, 1}>s a-a .
\end{array}\right.
$$

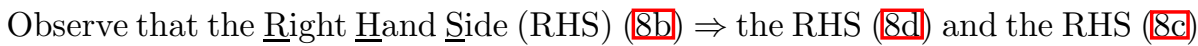
$\Rightarrow$ the RHS (8a). But the RHS (8b) holds for the condition $s \leqslant a^{-1} x_{p, 1}$ and the RHS (8d) also holds since $x_{p, 1} \leqslant a b \leq a^{n} \leqslant a^{n}+s a-a$. So, equation (17) is true and this implies that $G_{p}^{n}(x)=x_{p, 1}$ for every $x \in C$ completing the proof of (3b).

The proof of (BC) is quite similar. Now we take $x>x_{p, 1}$, which is equivalent to $s \geq a^{-1} x_{p, 1}+1$. This time we have to prove

$$
x_{p, 1}<A(s) \leq x_{p, 1}+1 \leq B(s)<x_{p, 1}+2
$$

for every $s \geq a^{-1} x_{p, 1}+1$. But now, we have the following equivalences

$$
\left\{\begin{array}{lll}
x_{p, 1}<A(s) & \Longleftrightarrow x_{p, 1}<s a \\
A(s) \leq x_{p, 1}+1 & \Longleftrightarrow \quad x_{p, 1} \geq s a-a^{n} \\
x_{p, 1}+1 \leq B(s) & \Longleftrightarrow \quad x_{p, 1} \leq s a-a \\
B(s)<x_{p, 1}+2 & \Longleftrightarrow \quad x_{p, 1}>s a-a^{n}-a .
\end{array}\right.
$$

In this occasion, the RHS (10b) $\Rightarrow$ the RHS (10d) and the RHS (10c) $\Rightarrow$ the RHS (10a). But the RHS (10b) holds since $x_{p, 1}+a^{n} \geqslant a^{n} \geqslant a b \geqslant s a$ and the RHS (10c) also holds for the condition $s \geqslant a^{-1} x_{p, 1}+1$. So, equation (91) is true and this implies that $G_{p}^{n}(x)=x_{p, 1}+1$ for every $x \in D$ completing the proof of (Bc). 
For (Bd), take $x \in B$ arbitrary, say, $x=(s-1) a+j$ as is shown in Table 1 . From [11, Théorème II.1.] it follows that

$$
C(s) \leqslant G_{p}^{n}(x) \leqslant D(s),
$$

where $C(s)=\frac{1}{a^{n-1}}\left(b y_{n}+s\right)$ and $D(s)=C(s)+1-\frac{1}{a^{n-1}}$.

Next, we need to demonstrate the four inequalities

$$
x_{p, 2}-1<C(s) \leqslant x_{p, 2} \leqslant D(s)<x_{p, 2}+1
$$

for every $s \in[1: b]$. And now, the equivalences are

$$
\left\{\begin{array}{lll}
x_{p, 2}-1<C(s) & \Longleftrightarrow \quad f(s)>a^{n-1}(1-j(p)) \\
C(s) \leq x_{p, 2} & \Longleftrightarrow \quad f(s) \leqslant a^{n-1}(a-j(p)) \\
x_{p, 2} \leq D(s) & \Longleftrightarrow \quad f(s) \geqslant a^{n-1}(1-j(p))+a-1 \\
D(s)<x_{p, 2}+1 & \Longleftrightarrow \quad f(s)<a^{n-1}(a-j(p))+a-1,
\end{array}\right.
$$

where $f(s)=s(a-1)-b(p-1)$, but the RHS (13b) $\Rightarrow$ the RHS (13d) and the RHS (13C) $\Rightarrow$ the RHS (113a). Then, we have to show the double inequality

$$
a^{n-1}(1-j(p))+a-1 \leqslant f(s) \leqslant a^{n-1}(a-j(p)),
$$

for every $s \in[1: b]$. For this, it is enough to prove these two inequalities

$$
a^{n-1}(1-j(p))+a-1 \leqslant f(1) \quad \text { and } \quad f(b) \leqslant a^{n-1}(a-j(p))
$$

and both are true since $b \leqslant \frac{a^{n-1}}{a-2}$. Thus, equation (12) holds and it implies that $G_{p}^{n}(x)=x_{p, 2}$ for every $x \in B$ completing the proof of (3d).

In order to prove equations (4a) and (4b), we only need to prove that equations (8c) and (10b) keep true if $n$ is replaced by $n-1$. The first is true because $x_{p, 1} \leq(b-1) a=a^{n-1} \leqslant a^{n-1}+s a-a$, and the second since $\frac{x_{p, 1}+a^{n-1}}{a} \geqslant$ $\frac{a+a^{n-1}}{a}=1+a^{n-2}=b \geqslant s$.

Finally, to prove equation (15), everything done for proving equation (3d) works well for $n+1$ instead of $n$. But, $b$ satisfies the condition $\frac{a^{n-1}}{a-2}<b<a^{n-1}$ $(a>3)$. Thus, $b \leq \frac{a^{n}}{a-2}$ and this condition guarantees the veracity of formula (5i), and the proof is done.

Remark 10. In [4. Theorem 4] was proved that the point $\frac{p q+1}{2}$ is a stable fixed point of $h$ the generalized TPT for a deck of $p q$ cards where $p, q \geq 3$ are odd integers. Well, from Theorem 8(b) and Theorem 9 equations (3d) and (5) follows the same result.

The minimum value of $k$ for which the power $G_{p}^{k}$ is a fixed point is not necessarily the one given in Theorem 9. To see this, just take $a=4, b_{1}=13$ and $b_{2}=14$. 
For $p=3$ Theorem 9 says that $n=3$ and $G_{3}^{4} \equiv 35$ for $b_{1}$ and $G_{3}^{4} \equiv 38$ for $b_{2}$ since $4<13<14<4^{2}$ and $14>13>\frac{4^{2}}{4-2}=8$. In this particular situation the minimum value is 3 in the first case and 4 in the second.

For a better understanding on the behavior of the family $\left\{G_{1}, \ldots, G_{a}\right\}$, based on Theorem 9, we present a couple of examples graphically.

Figure 2 shows the case $a=3, b=4$ for which $n=3$ and the fixed points are $1,6,7$, and 12. Observe that for $p=1,3$ three iterations are needed, otherwise just two.

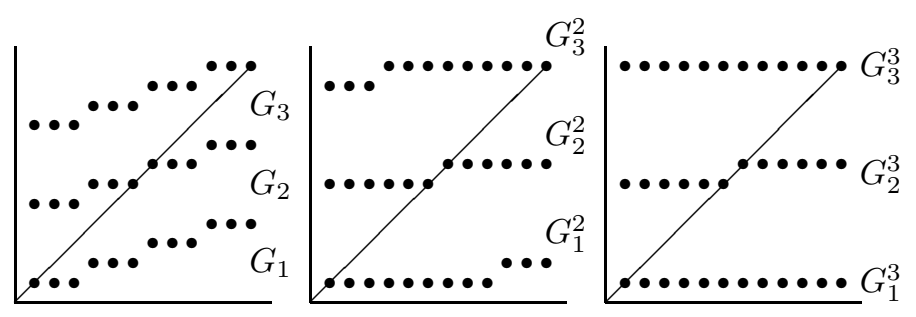

Figure 2: $a=3, b=4$.

Figures 3 and 4 show the case $a=4, b=14$. Again $n=3$, and the fixed points are $1,19,38$, and 56 . Figure 3 shows the graphics of $G_{p}$ and $G_{p}^{2}$, and Figure 4 the corresponding ones of $G_{p}^{3}$ and $G_{p}^{4}$. Observe that for $p=1,4$ three iterations are needed, otherwise four.

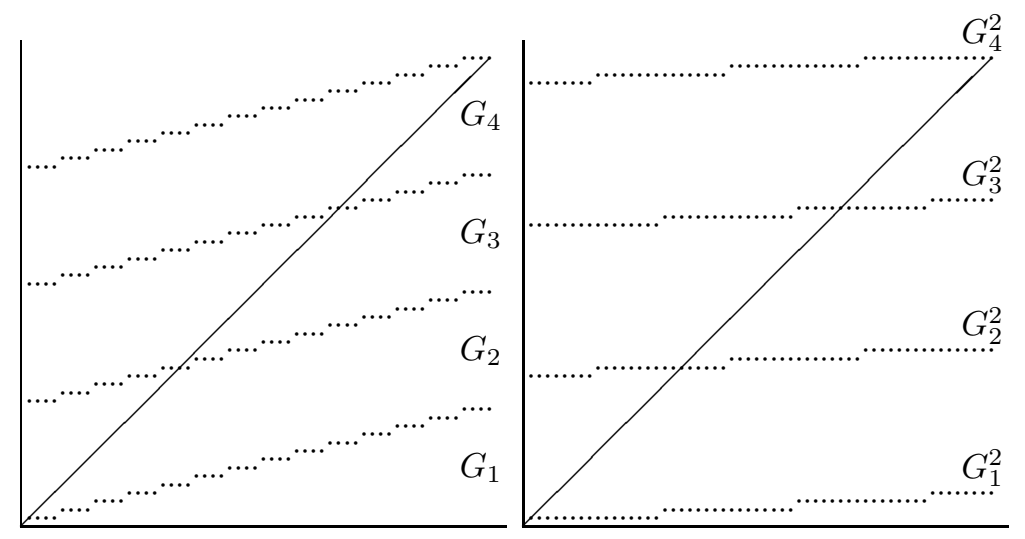

Figure 3: $a=4, b=14\left(G_{p}\right.$ and $\left.G_{p}^{2}\right)$. 


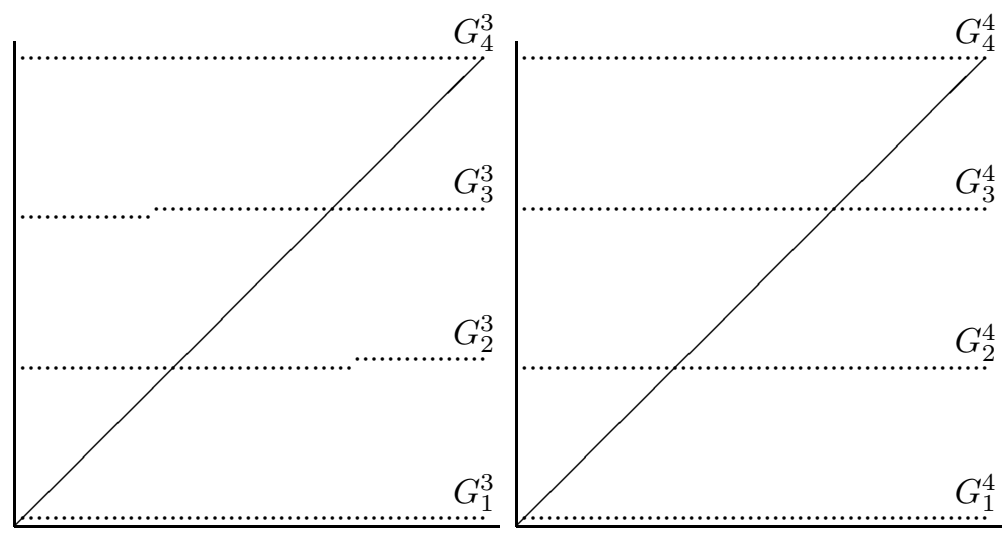

Figure 4: $a=4, b=14\left(G_{p}^{3}\right.$ and $\left.G_{p}^{4}\right)$.

\section{A bunch of brand new TPT-type card tricks}

Based on Theorems 8 and 9 we are in a position of generating some brand new TPT-type card tricks. For magicians some reasonable deck's sizes and performable with a portion or the whole pack of regular playing cards are those with $a b \in[8: 52](3 \leqslant a \leqslant 7)$. The 49 possible such decks and the 222 TPT-type variants of tricks are shown at Table 2 . Let us see the case $a=4, b=13$.

Example 11. (Performing protocol) Previously, the "magician" has to choose the value of $p$, say $p=3(1 \leqslant p \leqslant a)$. Then, she looks at Table 2 to get $n=3$ and the fixed point 35. Now, by the comment after Theorem 9, she knows that $G_{3}^{3}(x)=35$ for every $x \in[1: 52]$. Immediately, she has to perform the following general procedure:

Start with the cards downwards. Ask somebody from the audience to remember one of the cards without revealing it. Deal the $52(=a b)$ cards face up in $4(=a)$ rows, making 4 piles each $13(=b)$ high. After dealing, ask in which pile the card is. Take up the 4 piles, placing that pile $3^{\text {rd }}\left(=p^{\text {th }}\right)$ from the bottom. Turn the packet over. Do this twice more and count out the packet to the $35^{\text {th }}\left(=x_{p, 2}^{\text {th }}\right)$ card and turn it over to your audience's amazement and ovation.

Suppose the magician chooses $p$ for which $G_{p}$ has two consecutive fixed points, i.e., $x_{p, 1}, x_{p, 1}+1$. Once she picks up the cards for the last time and counts out the packet to the $x_{p, 1}^{t h}$ card she does not know whether it is the spectator's or next. To solve this situation she may add some patter at this moment and puts both cards together back to back and holds them with two fingers without showing them. Then, she asks the spectator: is this your card? while showing one. If the answer is yes, everything is done, otherwise she turns her hand and says here is your card and receives anyway her audience's applause. 
Table 2: The 49 decks and the 222 TPT-type variants

\begin{tabular}{|l|l|l|l|l|}
\hline$a$ & $b ; n$ & fixed points for each $p$ & $b ; n$ & fixed points for each $p$ \\
\hline 3 & $3 ; 2$ & $1-5-9$ & $4 ; 3$ & $1-6,7-12$ \\
\hline 3 & $5 ; 3$ & $1-8-15$ & $6 ; 3$ & $1-9,10-18$ \\
\hline 3 & $7 ; 3$ & $1-11-21$ & $8 ; 3$ & $1-12,13-24$ \\
\hline 3 & $9 ; 3$ & $1-14-27$ & $10 ; 4$ & $1-15,16-30$ \\
\hline 3 & $11 ; 4$ & $1-17-33$ & $12 ; 4$ & $1-18,19-36$ \\
\hline 3 & $13 ; 4$ & $1-20-39$ & $14 ; 4$ & $1-21,22-42$ \\
\hline 3 & $15 ; 4$ & $1-23-45$ & $16 ; 4$ & $1-24,25-48$ \\
\hline 3 & $17 ; 4$ & $1-26-51$ & & \\
\hline \hline 4 & $2 ; 2$ & $1-3-6-8$ & $3 ; 2$ & $1-4,5-8,9-12$ \\
\hline 4 & $4 ; 2$ & $1-6-11-16$ & $5 ; 3$ & $1-7-14-20$ \\
\hline 4 & $6 ; 3$ & $1-8-16-24$ & $7 ; 3$ & $1-10-19-28$ \\
\hline 4 & $8 ; 3$ & $1-11-22-32$ & $9 ; 3$ & $1-12,13-24,25-36$ \\
\hline 4 & $10 ; 3$ & $1-14-27-40$ & $11 ; 3$ & $1-15-30-44$ \\
\hline 4 & $12 ; 3$ & $1-16,17-32,33-48$ & $13 ; 3$ & $1-18-35-52$ \\
\hline \hline 5 & $2 ; 2$ & $1-3-5,6-8-10$ & $3 ; 2$ & $1-4-8-12-15$ \\
\hline 5 & $4 ; 2$ & $1-5,6-10,11-15,16-20$ & $5 ; 2$ & $1-7-13-19-25$ \\
\hline 5 & $6 ; 3$ & $1-8-15,16-23-30$ & $7 ; 3$ & $1-9-18-27-35$ \\
\hline 5 & $8 ; 3$ & $1-10,11-20,21-30,31-40$ & $9 ; 3$ & $1-12-23-34-45$ \\
\hline 5 & $10 ; 3$ & $1-13-25,26-38-50$ & & \\
\hline \hline 6 & $2 ; 2$ & $1-3-5-8-10-12$ & $3 ; 2$ & $1-4-8-11-15-18$ \\
\hline 6 & $4 ; 2$ & $1-5-10-15-20-24$ & $5 ; 2$ & $1-6,7-12,13-18,19-24,25-30$ \\
\hline 6 & $6 ; 2$ & $1-8-15-22-29-36$ & $7 ; 3$ & $1-9-17-26-34-42$ \\
\hline 6 & $8 ; 3$ & $1-10-20-29-39-48$ & & \\
\hline \hline 7 & $2 ; 2$ & $1-3-5-7,8-10-12-14$ & $3 ; 2$ & $1-4-7,8-11-14,15-18-21$ \\
\hline 7 & $4 ; 2$ & $1-5-10-14,15-19-24-28$ & $5 ; 2$ & $1-6-12-18-24-30-35$ \\
\hline 7 & $6 ; 2$ & $1-7,8-14,15-21,22-28,29-35,36-42$ & $7 ; 2$ & $1-9-17-25-33-41-49$ \\
\hline
\end{tabular}

Acknowledgements: I thank Dr. Kasturi Viswanath for proposing me "a question of interest" from which emerged this article.

\section{References}

[1] Bachet, C. G. Problèmes plaisans et délectables, qui se font par les nombres, partie recueillis de divers autheurs, et inventez de nouveau, avec leur démonstration, par Claude Gaspar Bachet, Sr. de Méziriac. Très utiles pour toutes sortes de personnes curieuses qui se servent d'arithmétique (1612). Edition consulted: C. Bachet, Problèmes plaisants et délectables qui se font par les nombres, Gauthier-Villars, Paris, 1884.

[2] Ball, W. W. R., Coxeter, H. S. M. Mathematical Recreations and Essays and Problems of Past and Present Times, Macmillan, London \& New York, 1892.

[3] Bolker, E. "Gergonne's card trick, positional notation, and radix sort", Mathematics Magazine, 83, 46-49, 2010. 
[4] Champanerkar, J., Jani, M. "Stable Fixed Points of Card Trick Functions", arXiv:1308.3396v1[math.HO], 1-10, 2013.

[5] Dickson, L. "Gergonne's pile problem", Bull. Amer. Math. Soc., 1, 184-186, 1895.

[6] Gardner, M. Mathematics Magic and Mystery, Dover Publications Inc., Mineola, N. Y., 1956.

[7] Gergonne, J. D., "Récréations Mathématiques: Recherches sur un tour de cartes", Annales de Mathématiques Pures et Appliquées, 4, 276-283, $1813-1814$.

[8] Harrison, J., Brennan, T., Gapinski, S. "The Gergonne p-pile problem and the dynamics of the function $x \mapsto\lfloor(x+r) / p\rfloor$ ", Discrete Applied Mathematics, 82, 103-113, 1998.

[9] Hudson, C. T. "Solution of question 2594", Educational Times Reprints, 9, 89-91, 1868.

[10] Hugard, J. Encyclopedia of card tricks, Faber and Faber, London, 1937.

[11] Quintero, R., Gerini, C. "Le «tour de cartes $\gg$ de Gergonne", Quadrature, $78,8-17,2010$.

[12] Quintero, R. "Un algoritmo basado en el formalismo de aplicación-código", 2do. Congreso Venezolano de Ciencia, Tecnología e Innovación, 7th-10th November, Caracas, Venezuela, 2013. 